\title{
Freak observers and the measure of the multiverse
}

\author{
Alexander Vilenkin \\ Institute of Cosmology, Department of Physics and Astronomy, \\ Tufts University, Medford, MA 02155, USA
}

\begin{abstract}
I suggest that the factor $p_{j}$ in the pocket-based measure of the multiverse, $P_{j}=p_{j} f_{j}$, should be interpreted as accounting for equilibrium de Sitter vacuum fluctuations, while the selection factor $f_{j}$ accounts for the number of observers that were formed due to non-equilibrium processes resulting from such fluctuations. I show that this formulation does not suffer from the problem of freak observers (also known as Boltzmann brains).
\end{abstract}




\section{INTRODUCTION}

The simplest interpretation of the observed accelerated expansion of the universe is that it is driven by a constant vacuum energy density, $\rho_{v}=$ const, which is about 3 times greater than the density of nonrelativistic matter. Ordinary matter is being diluted, while the vacuum energy density remains the same, and in another 10 billion years or so the universe will be compltely dominated by the vacuum. The following evolution of the universe is accurately described by de Sitter space.

It has been shown by Gibbons and Hawking [1] that the state of quantum fields in de Sitter space is similar to a thermal state with a characteristic temperature $T_{G H}=H / 2 \pi$, where

$$
H=\left(8 \pi G \rho_{v} / 3\right)^{1 / 2}
$$

is the de Sitter expansion rate. For the observed value of $\rho_{v}$, the Gibbons-Hawking temperature is extremely low, $T_{G H} \sim 10^{-29} \mathrm{~K}$. Nevertheless, interesting things will occasionally pop out of the vacuum as quantum fluctuations, at a nonzero rate per unit spacetime volume. An intelligent observer, like a human, could be one such thing. Or, short of a complete observer, a disembodied brain may fluctuate into existence, with a pattern of neuron firings creating a perception of being on Earth and observing the CMB radiation. Of course, the nucleation rate $\Gamma_{F}$ of such freak observers (also known as Boltzmann brains [2, 3, 4]) is extremely small [5, 6, 7]. But the important point is that it is nonzero.

De Sitter space is eternal to the future, so no matter how small $\Gamma_{F}$ is, freak observers will eventually outnumber regular observers who have ever lived in the universe [8, 9, 10]. Regular observers are formed as a result of non-equilibrium processes which started at the big bang and will eventually end when the universe thermalizes at the temperature $T_{G H}$. The total number of such observers that will exist in a fixed comoving volume is finite. On the other hand, the cumulative number of freak observers grows unboundedly with time. (In fact, it grows exponentially, since the corresponding physical volume grows as $\exp (3 H t)$.) Then the question is: Why are we not freak observers? (Assuming that we believe we are not.)

This issue has been recently discussed by Page [9], who concluded that the least unattractive way for us to avoid being freaks is to require that our vacuum should be rather unstable and should decay within a few Hubble times of the vacuum domination, that is, in 20 billion years or so.

Before accepting such a drastic conclusion, we need to analyze the situation in some more detail. Two important facts that need to be taken into account are (i) that our local universe appears to be a product of cosmic inflation and (ii) that inflation is generically eternal. Then bubbles of high-energy false vacuum can nucleate in our low-energy vacuum [12]. Such bubbles become sites of eternal inflation, producing an infinite number of pocket universes like ours, each containing an infinite number of observers [13]. The nucleation rate of false vacuum bubbles may be much lower that that of freak observers. But considering that each bubble nucleation yields an infinite number of regular obsevers, one might conclude that regulars totally outnumber the freaks [14].

The trouble is that in an eternally inflating universe the numbers of both types of observers are infinite. They can be meaningfully compared only if one adopts some prescription to regulate the infinities. A related issue, which has recently attracted much attention, is the calculation of probabilities for different vacua in multiverse models, also known as the measure problem. A number of prescriptions have been proposed, and one can try to apply 
them to the problem of freak observers. A "holographic" measure [15], which restrict consideration to the part of the universe within the causal diamond of a single observer, can resolve the problem if our vacuum is sufficiently unstable, enough for the vacuum decay to prevent freak domination [10]. Some ways to avoid the problem using a measure based on a globally defined time coordinate have been discussed in [11, 17].

Here, I am going to address the problem of freak observers in the context of the pocketbased measure, which was introduced in [16] and which satisfies the physically reasonable requirements of gauge-invariance and independence of initial conditions (see also [18]). In a recent paper, Bousso and Freivogel argued that this measure predicts freak domination and should therefore be ruled out. In fact, the formulation of the measure prescription in [16] disregarded the existence of freaks and was therefore incomplete. With freaks taken into account, the prescription as it stands gives meaningless infinite answers for the probabilities. I am going to suggest how the problem can be fixed by clarifying the formulation of the pocket-based measure.

\section{THE POCKET-BASED MEASURE}

The pocket-based prescription for the measure is a two-step procedure. The probability $P_{j}$ for a randomly picked observer to be in a pocket of type $j$ is given by the product

$$
P_{j}=p_{j} f_{j}
$$

where $p_{j}$ is an abundance of bubbles (pockets) of type $j$ and $f_{j}$ is the selection factor characterizing the relative number of observers in different pockets. To calculate the bubble abundance $p_{j}$, one first chooses a future-directed congruence of geodesics and a segment of a spacelike hypersurface $\Sigma$ which is crossed by that congruence. The geodesics project bubbles in the future of $\Sigma$ back onto $\Sigma$, and the prescription of [16] is to find what fraction of all bubbles is of type $j$, counting only bubbles whose projected size is greater than $\epsilon$, and then take the limit $\epsilon \rightarrow \infty$,

$$
p_{j}=\lim _{\epsilon \rightarrow 0} \frac{N_{j}(>\epsilon)}{N(>\epsilon)} .
$$

The resulting $p_{j}$ are independent of the choice of the geodesic congruence and of the hypersurface $\Sigma$. The bubble count is dominated by the bubbles nucleating in the asymptotic future, so the result is independent of the initial conditions at the onset of inflation. (An equivalent prescription for $p_{j}$ has been suggested in [19].)

The prescription for the selection factor $f_{j}$ is that it is given by the total number of independent observers that evolve in a fixed comoving volume,

$$
f_{j} \propto R^{3} \int_{\tau_{i n}}^{\infty} n_{j}(\tau) a_{j}^{3}(\tau) d \tau .
$$

Here, $R$ is a fixed comoving length scale, the same for all bubbles, $n_{j}(\tau)$ is the average number of observers produced in a pocket $j$ per unit physical volume per unit time and $a_{j}(\tau)$ is the scale factor in that pocket. The time coordinate $\tau$ is the proper time in the standard open FRW coordinates inside the bubble. The initial time $\tau_{\text {in }}$ is arbitrary, as long as it is chosen small enough. (At small $\tau$ all bubble spacetimes are identical, with $a(\tau)=\tau$.) Of course, the FRW bubble universes are infinite, and we could take the limit $R \rightarrow \infty$. But 
the constant factor $R^{3}$ drops out of the relative probabilities, so the value we choose for the length scale $R$ is unimportant.

In all bio-friendly bubbles, there should be a period of internal inflation, characterized by a large expansion factor $Z_{j} \gg 1$. After the vacuum energy is thermalized, a certain number $\mathcal{N}_{j}^{*}$ of observers will evolve per unit thermalized volume; its value will depend on the local parameters of the low-energy physics. Counting only regular observers, as it was done in [16], we can write

$$
f_{j} \sim Z_{j}^{3} \mathcal{N}_{j}^{*}
$$

But now we know that if the vacuum energy is positive inside the bubble, then, apart from the regular observers, there are freak observers who nucleate at a constant rate per unit spacetime volume. This means that, if the freaks are included, then $n_{j}(\tau) \rightarrow$ const and Eq. (4) gives $f_{j} \rightarrow \infty$. Moreover, as already mentioned in the Introduction, bubbles of false vacuum will also nucleate at a constant rate, each bubble contributing infinite numbers of both the regulars and the freaks.

Clearly, the prescription (44) is not acceptable as it stands. The intent of the original formulation in [16] was to count only regular observers who evolve in the wake of bubble nucleation. But the question is: On what basis can we discriminate against the freak observers? It is not enough to say that they are formed by quantum fluctuations. In models of cosmic inflation, galaxies and other cosmic structures owe their eistence to quantum fluctuations, so human observers may share the fuzzy quantum origin with the freaks. In the next Section I will suggest a possible way of regulating the infinity in Eq. (44).

\section{GETTING RID OF FREAK OBSERVERS}

My proposal is that there should be a sharp division between the kinds of objects counted in $p_{j}$ and those counted in $f_{j} . p_{j}$ counts the objects like bubbles, which nucleate in a vacuum at a constant rate. These are equilibrium vacuum fluctuations in de Sitter space. $f_{j}$ counts observers that arise due to non-equilibrium processes in the wake of a quantum fluctuation of type $j$. This can still be expressed by Eq. (4) if we make the replacement

$$
n_{j}(\tau) \rightarrow \tilde{n}_{j}(\tau)=n_{j}(\tau)-n_{j}^{(e q)} .
$$

Here, $n_{j}(\tau)$ is the total production rate of observers, $n_{j}^{(e q)}=$ const is the equilibrium rate at which they are produced by quantum fluctuations in a de Sitter vacuum, and the difference $\tilde{n}_{j}(\tau)$ is the production rate due to non-equilibrium processes. With this replacement, the integral in (44) is convergent and can still be estimated by Eq. (5).

To put it slightly differently, the events counted in $p_{j}$ are uncaused, random, equilibrium fluctuations. The factor $f_{j}$ assigns a weight to these fluctuations, based on the average number of observers formed as a result of non-equilibrium processes caused by a fluctuation of type $j$. A fluctuation producing one isolated freak observer gets a weight of 1 . Even if there is a huge fluctuation producing a large number of freaks, the weight will always be finite. On the other hand, a bio-friendly bubble produces an infinite number of observers and thus has an infinite weight. Formally, this can be accounted for by taking the limit $R \rightarrow \infty$ in Eq. (4). As a result, freak observers get a vanishing relative weight, while the relative weights of the bubbles are independent of $R$. 


\section{DISCUSSION}

The purpose of this note is to clarify the pocket-based measure (21) of Ref. [16]. My proposal is that the factor $p_{j}$ should be interpreted as the abundance of equilibrium vacuum fluctuations of a given type. The selection weight $f_{j}$ is proportional to the average number of observers formed due to non-equilibrium processes resulting from such fluctuations. Freak observers are produced either individually or in finite groups, while each bio-friendly bubble produces an infinite number of observers. Thus, freak observers have a vanishing relative weight and do not contribute to the measure, even though their nucleation rate may in some cases be higher than that of the bubbles.

I should finally mention some open issues. The pocket-based measure, as it is presently formulated, assumes that bubbles do not collide with one another. Also, the current formulation cannot be directly applied to models where pockets are formed by quantum diffusion. Some ideas toward extension to this class of models have been discussed in [16].

The pocket-based measure does account for bubble formation within bubbles. However, it is tacitly assumed that secondary bubbles ( $s$-bubbles) do not interfere with the evolution of observers in the primary bubble ( $p$-bubble). This should be a good approximation if the $s$-bubble formation rate is very low. The production rate of observers in Eq. (6) approaches zero when the stars die out and other non-equilibrium processes in the $p$-bubble come to a halt. We assume that $s$-bubbles that nucleate during the period when $\tilde{n}_{p}(\tau)$ is substantially different from zero affect only a small fraction of volume in the open FRW universe of the $p$-bubble. Inclusion of this effect should result in a slight renormalization of the selection factor $f_{p}$.

The formation rate of freak observers is likely to be enhanced for some period of time $\tau$ inside the bubbles, either due to thermal fluctuations (while the temperature is still higher than $\left.T_{G H}\right)$ or to quantum fluctuations induced by time-varying fields and other non-equilibrium processes in the wake of bubble nucleation. This is good news for the freaks: their formation rate, given by Eq. (6) , is non-zero after all. However, just as in the case of regular observers, this rate approaches zero at large $\tau$, and the fraction of freaks relative to the regular observers is likely to be very small. The nucleation rate of $s$-bubbles may also be enhanced at early times. This will probably result in some additional renormalization of $p_{s}$ and $f_{p}$. This issue requires further study.

\section{Acknowledgments}

My views on these matters have been shaped in numerous discussions with Jaume Garriga. I am also grateful to Raphael Bousso, Ben Freivogel and Roni Harkin for stimulating discussions during my visit to Berkeley. This work was supported in part by grant RFP106-028 from The Foundational Questions Institute.

[1] G.W. Gibbons and S.W. Hawking, Phys. Rev. D15, 2738 (1977).

[2] To my knowledge, Martin Rees [3] was the first to discuss disembodied brains arising from thermal or quantum fluctuations, in connection with the Boltzmann's idea that we are observing a huge fluctuation in the otherwise thermalized universe. The term "Boltzmann's 
brains" was later coined by Albrecht and Sorbo [4], who also discussed the issue in relation to inflationary cosmology.

[3] M.J. Rees, Before the Beginning, p.221 (Addison-Wesley, 1997).

[4] A. Albrecht and L. Sorbo, Phys. Rev. D70, 063528 (2004); see also A. Albrecht, in Science and Ultimate Reality, ed. by J.D. Barrow, P.C.W. Davies and C.L. Harper (Cambridge University Press, 2003).

[5] L. Dyson, M. Kleban and L. Susskind, JHEP 0210, 011 (2002).

[6] D.N. Page, J. Corean Phys. Soc. 49, 711 (2006).

[7] D.N. Page, hep-th/0610199.

[8] L. Susskind, as quoted in [7]; see also [5].

[9] D.N. Page, arXiv:hep-th/0610079.

[10] R. Bousso and B. Freivogel, arXiv:hep-th/0610132.

[11] A.D. Linde, arXiv:hep-th/0611043.

[12] K. Lee and E.J. Weinberg, Phys. Rev. D36, 1088 (1987).

[13] J. Garriga and A. Vilenkin, Phys. Rev. D57, 2230 (1998).

[14] This argument has been independently suggested by Andrei Linde, private communication.

[15] R. Bousso, hep-th/0605263.

[16] J. Garriga, D. Schwartz-Perlov, A. Vilenkin and S. Winitzki, JCAP 0601, 017 (2006).

[17] D.N. Page, hep-th/0611158.

[18] A. Vilenkin, arXiv:hep-th/0609193.

[19] R. Easther, E.A. Lim and W.R. Martin, JCAP 0603, 016 (2006). 